\title{
Acoso y hostigamiento sexual en el ámbito universitario, los caminos de la práxis
}

\author{
Abuse and sexual harassment in the university context, the ways of práxis
}

Abuso e assédio sexual no contexto universitário, os caminhos da práxis

Maria Jose Garcia Oramas ${ }^{2}$ ip

\begin{abstract}
Resumen: En este trabajo se analiza la implementación de un programa de prevención, atención y sanción del acoso y el hostigamiento sexual en la Universidad Veracruzana (UV), a partir de indagatorias realizadas entre las y los integrantes de la comunidad universitaria y a la luz de las teorías de género. Se enfatiza en la implicación de la autora en este proceso concebido como una praxis donde, si bien ha sido difícil mantener una distancia óptima frente a la problemática de la violencia de género, es precisamente a partir de la experiencia vivida como han surgido las propuestas para transformarla, inmersa en un contexto tan hostil como lo es el México actual y especialmente el Estado de Veracruz, donde los índices de violencia contra las mujeres y las altas tasas de feminicidio afectan directamente la vida de toda la población, incluidas estudiantes y trabajadoras de esta casa de estudios. Se postula que frente a la violencia ha de imponerse, necesariamente, el diálogo y la palabra generadora de sentido y, por ende, las y los distintos actores de la comunidad universitaria han de interactuar de manera colectiva para desmontar las estructuras jerárquicas, androcéntricas y de dominación masculina vigentes.
\end{abstract}

Palabras clave: Universidad. Acoso. Hostigamiento sexual.

Resumo : O presente trabalho analisa a implementação de um programa de prevenção, atenção e punição ao assédio e assédio sexual na Universidad Veracruzana $(U V)$, baseado em inquéritos realizados entre os membros da comunidade universitária à luz das teorias de gênero. Enfatiza-se a implicação da autora no processo, concebido como uma práxis em que, embora tenha sido difícil manter uma distância frente à problemática de violência de gênero, foi precisamente a partir da experiência vivida que propostas surgiram para transformá-la. Experiência esta imersa em um contexto tão hostil como é hoje o México e especialmente o Estado de Veracruz, no qual as taxas de violência contra as mulheres e as altas taxas de feminicídio afetam diretamente as vidas de toda a população, incluindo estudantes e trabalhadoras desta universidade. Supõem-se que, diante da violência, o diálogo e a palavra geradora de sentido devem necessariamente serem sustentados e os diferentes atores da comunidade universitária devem interagir coletivamente para desmontar a estrutura hierárquica, androcêntrica e de dominação masculina atual.

Palavras-chave: Universidade. Assédio. Assédio sexual.

Abstract: Here I analyze the results of the implementation of a program designed to prevent and sanction abuse and sexual harassment at Universidad Veracruzana $(U V)$, based on survey conducted among members of the University's community. I use a gender theory framework, emphasizing the implication of the author in a process conceived as a praxis where despite the difficulty of keeping an optimal distance from the phenomenon of gender violence, I argue that it is precisely the lived experience that has informed the initiatives to transform it. This, in the midst of the prevailing climate of violence in Mexico and in the State of Veracruz as heightened levels of gender violence and incidence of femicides have affected the lives of the entire population, including those of University's students and employees. The proposed approach is to facilitate the collective interaction of all different members of the University's community to make sense, through dialogue, of the phenomenon of violence, as well as, to dismantle current hierarchical, androcentric and dominant masculine structures.

Keywords: University. Abuse. Sexual harassment.

\footnotetext{
${ }^{1}$ Enviado el: 27 Nov. 2018 - Aceptado el: 16 Mayo 2019 - Publicado el: 19 Nov. 2019

${ }^{2}$ Universidad Veracruzana (Mexico) - Email: maria_josegarcia@hotmail.com
} 
Imagen 1. Instalación por la paz com estudiantes de la Facultad de Danza de la Universidad Veracruzana, realizada por Fernán González

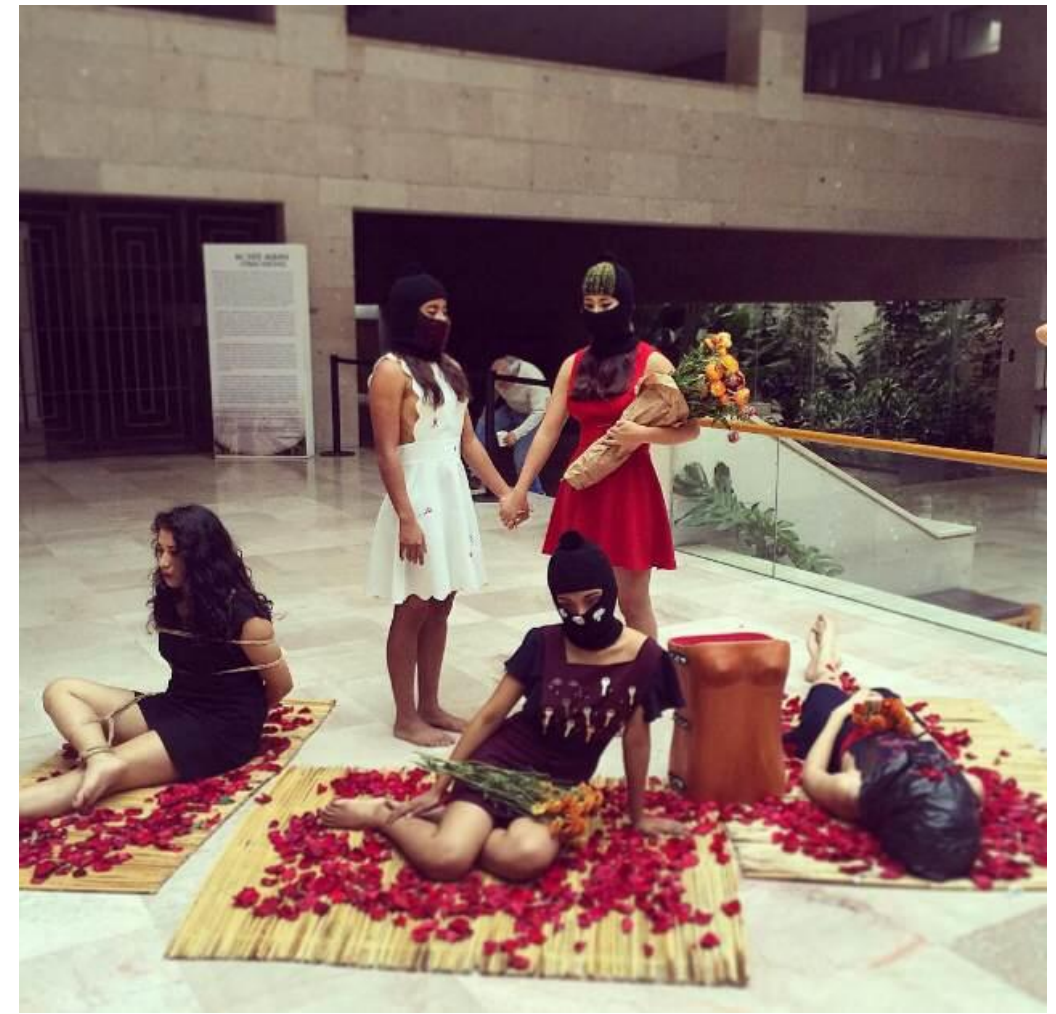

Fuente: Universo - Sistema de noticias de la UVi: "Violencia contra las mujeres aún persiste".

\section{Ser mujer en la universidad}

Nacer mujer es llegar a este mundo en situación de desventaja. Alrededor del planeta y en pleno siglo XXI, las niñas siguen contando con menos posibilidades de ser educadas, alimentadas, incluso escogidas para sobrevivir si el número de hijos permitidos para cada pareja es limitado. Además, tienen más posibilidades de ser violentadas, secuestradas, explotadas laboral y sexualmente e incluso de ser vendidas o intercambiadas por otros bienes. A partir de los 10 años las niñas empiezan ya a convertirse en madres ${ }^{\mathrm{ii}}$ y la pobreza se va reproduciendo inter-generacionalmente a causa de la baja escolarización, las altas tasas de desempleo y la mayor precariedad laboral que afectan a la mayoría de la población mundial y, en mayor medida, a las mujeres, lo que da como resultado el que las más pobres entre los pobres sean, invariablemente, niñas y más específicamente, niñas indígenas.

Ser mujer y llegar a la universidad es pues un privilegio al que pocas mujeres pueden acceder y, mientras en algunos países aún se les prohíbe estudiar, en México, si bien el derecho a la educación es universal, únicamente 21 de cada 100 niños que inicia la escuela primaria termina la universidad sea por falta de recursos económicos para comprar útiles escolares, pago de pasajes y de inscripciones. De ellos, menos de la mitad serán mujeres (OCDE, 2018).

Y, haciendo historia, la admisión de las mujeres a la educación superior, de manera formal, tardó 8 siglos más que para los varones si consideramos que la universidad más antigua 
en Europa se fundó en Bolonia, Italia, en el siglo XII y las mujeres no fueron admitidas hasta 1860 en Suiza; hacia 1870 en Inglaterra; en 1880 en Francia; y hasta 1900 en Alemania. En México, la primera mujer graduada en la universidad fue la médica, Matilde Montoya, quien se tituló en 1887. En 1889 se tituló la primera mujer abogada, María Asunción Sandoval (GARCÍA, 2018).

Es indudable que en nuestros días hay avances significativos en el acceso de las mujeres a la educación superior puesto que en el último siglo la incorporación de las mujeres a la Universidad creció de manera exponencial y la población femenina en las aulas a nivel mundial pasó de un tercio de la matrícula total, en 1960, a casi la mitad, en 1995, hasta llegar al 50 por ciento en el $2000 \mathrm{y}$, que esta tendencia se mantiene en rápido crecimiento desde entonces (ONU, 2011). Sin embargo, la creciente representación de las mujeres en la educación superior no ha impactado, aún, en su participación sustantiva en el desarrollo científico, mientras las brechas de género y la violencia en su contra persisten en las universidades.

A las dificultades económicas para ejercer la actividad científica en México hay que sumar entonces las brechas de género, entendidas como los aspectos puntuales en donde las desigualdades son mayúsculas limitando la participación de las mujeres. Los datos de participación femenina dedicadas a la actividad científica muestran un porcentaje significativamente menor al de varones, con una proporción de una mujer por cada tres varones (ONU, 2011) y ello en un sector caracterizado por la falta de inversión, misma que ha ido disminuyendo de manera continua en las dos últimas décadas en detrimento del desarrollo científico, particularmente en las universidades del sector público que cuentan cada día con menor presupuesto y mayor demanda.

Los modelos androcéntricos y los prejuicios y estereotipos de género que permean nuestras sociedades son un factor preponderante en la persistencia de las desigualdades e inciden en la segregación horizontal y vertical en las universidades. Ello se traduce en que la mayoritaria presencia de las mujeres en la universidad todavía no impacte en su participación representativa en los puestos de autoridad y de toma de decisiones. Más aún, que en las universidades continúen existiendo desigualdades interseccionales que se asocian también con condiciones de etnia y clase social, lo que dificulta el pleno goce de los derechos de las mujeres; sobre todo, entre quienes pertenecen a distintas etnias y a sectores menos favorecidos en nuestra sociedad $^{\mathrm{iii}}$ (segregación vertical), ni en su distribución paritaria en las distintas esferas del conocimiento (segregación horizontal).

\section{Violencia de género en la universidad y políticas de prevención, atención y sanción de casos de acoso y hostigamiento sexual: el caso de la universidad veracruzana.}

En materia de violencia de género, la vida cotidiana de las mujeres en las universidades transcurre, como en otros espacios, en medio de situaciones de acoso y hostigamiento sexual. Tan es así, que en 2017 el Comité de la Comisión para Eliminar la Discriminación y la Violencia Contra las Mujeres (CEDAW) hizo una recomendación específica al Estado 
mexicano para actuar en contra de este flagelo, dada la alta incidencia casos a nivel de educación media y superior, lo que corresponde a niveles de bachillerato, licenciatura y posgrado.

En el informe, titulado "De la violencia a la convivencia escolar en la educación media superior. Una aproximación con perspectiva de género", citado en la recomendación de la CEDAW (2018), se reporta que las estudiantes mexicanas de nivel medio superior identifican los espacios violentos en: el ámbito familiar (37\%), seguido del espacio escolar (31\%), docente (19\%) y la violencia en el noviazgo (13\%). Mientras que los estudiantes varones dicen que el ámbito escolar (51\%) resulta más violento, así como la comunidad (19\%) y el espacio docente (14\%). Asimismo, el informe indica que las conductas sufridas por las estudiantes son aquellas que las ridiculizan u ofenden (32\%), la intimidación y amenaza (17\%), así como las bromas hirientes y descalificaciones (17\%). De igual forma indica que los principales agresores identificados por las estudiantes son sus compañeros de escuela (26\%), quienes les ridiculizan, ofenden y llegan a realizar bromas hirientes afectándolas de forma psicoemocional, los y las profesores/as también son identificados/as dentro de la escuela como agresores/as.

La recomendación al Estado Mexicano especifica que: la Secretaría de Educación Pública del país carece de protocolos de atención a la violencia de género en las escuelas y en la educación y en general carece de una política articulada y sistemática en materia de prevención, atención, sanción y erradicación a la violencia de género en las instituciones educativas (CEDAW, 2018). Esta situación provocó que la Asociación Nacional de Universidades e Instituciones de Educación Superior (ANUIES), hiciera un llamado a todas las universidades del país afiliadas a contar con protocolos de acción para actuar en estos casos, recomendación que hasta el presente no se ha concretado en todos los casos.

En el caso de la Universidad Veracruzana, se creó la Coordinación de la Unidad de Género en 2014 y paralelamente, ese año un equipo de investigadores inició una investigación que se publicaría en 2017 bajo el título: "Estudios sobre la violencia de género en la Universidad" (CASILLAS et al. 2017). En esta obra se analiza la violencia de género en la universidad desde distintas perspectivas e incluye indagaciones a las y los estudiantes de nuestra casa de estudios. Una de las investigaciones (CASILLAS et al. 2017), recoge los resultados de una encuesta aplicada a estudiantes mediante un estudio de corte cuantitativo sobre representaciones sociales de la violencia de género, aplicado a una muestra representativa de 1931 estudiantes, hombres y mujeres, de las distintas áreas y regiones de la UV. Los resultados coinciden con el reporte del CEDAW ya mencionado y muestran que tanto en la escuela previa como en la Universidad, las y los encuestados han experimentado distintas formas de violencia de género, destacando que el principal agente agresor fueron los propios compañeros, profesores y autoridades escolares. El estudiantado comentó no estar de acuerdo en que la violencia docente sea algo normal y natural (85.6\%), y 8 de cada 10 estudiantes indicaron que este tipo de actos son indignantes, que les enoja y al mismo tiempo consideran que debe ser denunciado, aun cuando únicamente un $29.0 \%$ lo ha hecho.

En otra de las investigaciones incluida en la obra antes mencionada (JONGITUD ZAMORA, 2017) y llevada a cabo también con estudiantes, pero esta vez de corte cualitativo, se realizaron de manera aleatoria entrevistas a profundidad a 186 estudiantes, $47.7 \%$ fueron 
hombres y un 54.3\% mujeres. Se logró identificar que, desde la mirada de las y los estudiantes, "los hombres son violentos porque son más fuertes, porque obedecen al instinto natural", "es la testosterona la que hace que se hagan ese tipo de actos violentos". Atribuyen que ello se debe al "rol que nos da la sociedad", en donde se ha establecido que "las mujeres tenemos que ser más tranquilas, sumisas, calmadas y estar en la sombra", mientras que los hombres deben estar "al sol". Además, pudieron valorar que los profesores tienen ciertas "preferencias hacia las mujeres o hacia los hombres". Al parecer las docentes mujeres, prefieren a los hombres por sus características "guapo".

Las estudiantes mujeres son las que consideran que la violencia sexual proviene de los "maestros pervertidos, que se les quedan viendo a las alumnas y descaradamente" y esto les genera enojo e indignación. Reconocen que cuando estos maestros "captan", entonces "se hacen los que no veían nada". Las miradas son las que intimidan. En cuanto a la violencia sexual, las y los estudiantes consideran que si existe en la universidad y que se genera cuando hay "una chica guapa", y "el profesor la ve con ojos de que le quiere hacer cosas obscenas". La violencia sexual también logran verla como un conjunto de "comentarios despectivos o vulgares", denotaciones fuera del contexto, tocamientos del cabello o del cuerpo de manera involuntaria y sin el consentimiento, acercamientos al momento de saludar. Este tipo de violencia sexual, también la califican de "acoso". Al igual que en el estudio anterior, manifestaron no solicitar ayuda a las instancias oficiales y cuando lo hicieron fue con sus profesores o con autoridades universitarias pero consideraron que el impacto de la atención no fue significativo.

Finalmente, en su aportación sobre atención y prevención del acoso sexual, Guzmán Ibáñez (2017, p. 92) afirma:

dentro de las universidades hoy son endémicas las prácticas relacionadas al acoso de los varones hacia las mujeres, el más frecuente es el de los profesores hacia las alumnas, que integra simultáneamente las dos esferas de dominación: la jerarquización del poder y la de género, pero también se reproduce en los espacios de pares, en los que los compañeros de estudios hostigan sexualmente a las estudiantes.

La autora afirma que resulta evidente el hecho de que atacar este fenómeno implica actuar desde una variada cantidad de aristas que ameritan profundas indagaciones pero enfatizando que:

el silencio ante este tipo de prácticas es un elemento esencial para su perpetuación, tanto el silencio de las víctimas que se resisten a denunciar, pero también el de los espectadores, que prefieren resguardarse en el anonimato en vez de apoyar a las víctimas. La víctima duda en hablar porque en caso de denunciar el hecho no se otorguen las sanciones pertinentes o en el peor de los casos las represalias podrían afectar su tránsito académico por la misma complicidad existente entre autoridades y docentes, que en algunos casos simplemente lo dejarán passar (GUZMÁN IBÁÑEZ, 2017, p. 93).

A fin de dar respuesta institucional a esta lamentable situación, en Marzo del 2014 se crea por acuerdo rectoral la Coordinación de la Unidad de Género, mismo que fue ratificado por el Consejo Universitario General, máxima autoridad de la Universidad Veracruzana. Las autoridades universitarias propusieron a la autora de este texto como la primera coordinadora 
de esta dependencia. Acepté la propuesta y durante 4 años, período en que duró este encargo, diseñé e implementé las políticas institucionales encaminadas a promover una cultura de equidad de género en la Universidad, incluyendo la atención a casos de acoso y hostigamiento sexual y de no discriminación a personas con identidades sexo-genéricas diversas.

Para lograr los objetivos propuestos y con el apoyo de un pequeño equipo de trabajo, nos avocamos, en primera instancia, en generar una estructura organizacional que garantizara la transversalidad actuando desde una dependencia pequeña, pero vinculada estrechamente con las diversas entidades académicas y dependencias administrativas de la universidad, enfatizando la descentralización y asegurando que las acciones emprendidas se llevaran a cabo en las cinco las regiones en que se compone la universidad y en las cuatro sedes de la Universidad Veracruzana Intercultural (UVI) cuyos estudiantes son en su mayoría de origen indígena.

Conformamos el Comité de Igualdad de Género integrado por representantes de estas regiones y sedes de la UVI y con este grupo formulamos anualmente el Plan Institucional de Igualdad de Género y, alineado a este programa, pero respetando la diversidad regional, los Programas Regionales. Las y los representantes llevaron a cabo las actividades con el apoyo de enlaces, encargados de la promoción de la igualdad de género en todos los departamentos y entidades académicas. Finalmente, generamos el Consejo Consultivo para la Igualdad de Género, órgano colegiado de opinión, consulta y resolución, encargado de atender los asuntos relacionados con la igualdad de género en la UV.

Para generar un marco legal que nos permitiera actuar en casos de acoso y hostigamiento sexual, junto con un equipo de expertas creamos el "Reglamento para la Igualdad de Género de la Universidad Veracruzana" (UNIVERSIDAD VERACRUZANA, 2015b) y la "Guía para la atención de casos de acoso y hostigamiento sexual" (UNIVERSIDAD VERACRUZANA, 2015a). El Reglamento fue aprobado en diciembre del 2015 por el Consejo Universitario General luego de un proceso de revisión en distintas instancias universitarias y desde entonces constituye un lineamiento institucional de observancia obligatoria y generalizada, que establece las disposiciones normativas que regulan y aseguran los principios de igualdad y no discriminación por razones de sexo o género en nuestra Universidad.

En el capítulo II del Reglamento para la Igualdad de Género de la UV, relativo a la atención a denuncias relacionadas con la discriminación y la violencia por sexo o género, se establece lo siguiente:

Artículo 35. Cuando alguna persona integrante de la comunidad universitaria considere haber sufrido algún tipo de violencia de género o de discriminación por su condición sexogenérica, bajo la más estricta responsabilidad y mediante la presentación de elementos de prueba podrá interponer por escrito la denuncia o queja ante la autoridad correspondiente, siguiendo los procedimientos establecidos en la normatividad universitaria aplicable y en la Guía para la Atención de Casos de Hostigamiento y Acoso Sexual.

Artículo 36. Queda prohibido someter a las víctimas por violencia o discriminación a cualquier mecanismo de conciliación o mediación, antes o durante los procesos de investigación y sanción.

Artículo 37. Las autoridades unipersonales que conozcan de los casos de discriminación por sexo o género o los tipos de violencia en sus diferentes modalidades, deberán evitar la victimización de las personas a través de acciones 
adecuadas implementadas de oficio o bien a petición de la víctima, las cuales serán ejecutadas de conformidad con lo establecido en la normatividad universitaria (UNIVERSIDAD VERACRUZANA, 2015b, p. 20).

El Capítulo III, relativo a la erradicación de la discriminación sexo-genérica se establece lo siguiente:

\begin{abstract}
Artículo 38. Las autoridades unipersonales, en el ámbito de sus competencias y con base en la investigación realizada en la atención de casos de discriminación por sexo o género o violencia hacia las mujeres, deberán imponer las sanciones correspondientes que tengan por objetivo erradicar dichas prácticas.

Artículo 39. La víctima de violencia o discriminación en cualquier momento puede acudir ante la vía administrativa o judicial para ejercer su derecho a la justicia, sin que la presentación de las denuncias o quejas ante la Universidad constituyan un mecanismo alterno para ejercer los derechos que correspondan (UNIVERSIDAD VERACRUZANA, 2015b, p. 20-21).
\end{abstract}

Por su parte, "la Guía para la atención de casos de acoso y hostigamiento sexual" establece la hoja de ruta, es decir, el protocolo de acción para actuar en estos casos y consta de tres apartados: el primero contiene el marco conceptual incluyendo un glosario de términos; el segundo indica los elementos que caracterizan este tipo de actos en función de la normativa internacional, nacional, estatal y universitaria vigente y, el tercero explica el procedimiento para la atención a quienes hayan sido víctimas de alguna de estas situaciones.

En el glosario de términos de la guía se describe como acoso sexual al

comportamiento de carácter sexual en donde no existe la subordinación pues su actuar se da de manera horizontal, que no es deseado y resulta ofensivo para quien lo recibe, que puede implicar contacto físico e insinuaciones, observaciones de tipo sexual, exhibición de pornografía, exigencias sexuales, verbales o de hecho, que humillen y afecten la salud y la seguridad de la víctima (UNIVERSIDAD VERACRUZANA, 2015a, p. 23).

Por su parte, el hostigamiento sexual es

el comportamiento ofensivo, incómodo o humillante, que se desarrolla frente al ejercicio del poder en una relación de subordinación expresada a través de conductas verbales, no verbales y físicas, relacionadas con la sexualidad de connotación lasciva y que niega a las personas la dignidad, el respeto y la igualdad de trato a la que tienen derecho (UNIVERSIDAD VERACRUZANA, 2015a, p. 24).

En este sentido, jurídicamente, el hostigamiento y el acoso sexual son formas diferentes de violencia de género y ambos ejercen daños a la víctimas asociados a la exclusión, la subordinación, la discriminación y la explotación de las mujer y por ello son considerados como "comportamientos consubstanciales a la opresión de género en todas sus modalidades, afectando sus derechos humanos" (MEXICO, 2018). La diferencia entre uno y otro, es que mientras el hostigamiento sexual se produce en sentido vertical, es decir, existe un contexto de autoridad-subordinación ya sea en el ámbito laboral, como en el caso de autoridades y personal o, en el educativo, en el caso de autoridades y docentes o entre docentes y alumnos; el acoso sexual, por su parte, si bien se da en un contexto de relaciones horizontales, al ser no deseado resulta también ofensivo para quien lo recibe. 
Gracias a esta normativa, la Coordinación de la Unidad de Género logró brindar 43 asesorías jurídicas entre 2016 y 2017: 34 de ellas de su competencia y 9 fuera de ella que fueron canalizadas a otras dependencias universitarias. Se atendieron a 49 alumnas, 4 académicas, 5 trabajadoras del área administrativa, técnica y manual, 1 alumno y un grupo de estudiantes integrado por hombres y mujeres. De estas asesorías, 21 terminaron en denuncia, 15 por la vía universitaria y 6 por la vía penal y 13 sin denuncia. Las principales razones por las que no denunciaron coinciden con el reporte de las investigaciones realizadas en la universidad anteriormente mencionadas, y tuvieron que ver con el miedo y el temor a represalias y, en menor medida, a problemas de salud física o psicológica de las víctimas. De las 15 denuncias interpuestas en la Universidad, en 12 se dictaron sanciones, todas ellas a varones: académicos, estudiantes y trabajadores eventuales. Las sanciones iban desde la amonestación hasta la suspensión temporal y la recisión definitiva. En los 3 casos restantes no se dictó sanción por falta de elementos.

Cabe destacar que estas cifras correspondieron a las estadísticas que se han generado en la región, en el país y en los propios estudios llevados a cabo en la Universidad, esto es, al hecho de que la violencia de género en su gran mayoría tiene como víctima a una mujer y como victimario a un varón que no es un desconocido, sino alguien cercano a ella, en este caso, un docente, compañero de trabajo o compañero alumno. También, que las víctimas tienen miedo y temor a denunciar estos hechos porque ello puede generar represalias que pueden afectar su vida laboral o estudiantil pero que, en la medida en que se sienten acompañadas y apoyadas, se va ganando confianza en la institución y en la importancia de llevar a cabo los procedimientos de denuncia siempre apegados a la legislación universitaria.

Paralelamente a las acciones de atención de la violencia de género en la universidad, en materia de prevención se llevó a cabo la campaña \#elsilenciomarcatuvida, de carácter permanente y teniendo como ejes la difusión y capacitación del acoso y hostigamiento sexual entre las y los integrantes de la comunidad universitaria. En este marco de acción, durante 2016 y 2017 se capacitaron a todas y todos los funcionarios y autoridades de las entidades académicas en todas las regiones de la UV y sedes de la UVI, a fin de darles a conocer el Reglamento para la Igualdad de Género y también a integrantes de comités sindicales de docentes y de personal administrativo. La campaña se difundió a través de la proyección de videos, la distribución de pendones, dípticos, redes sociales y materiales diversos y para todo ello se contó con el apoyo financiero de la Secretaría de Educación Pública Federal, a través del programa de Fortalecimiento a la Calidad de la Educación para la Igualdad de Género (PFCE).

Finalmente, es relevante mencionar que la Universidad Veracruzana fue una de las primeras universidades en el país en contar con herramientas legales y en actuar a favor de una vida libre de violencia contra las mujeres en sus campus y, por ello, las políticas emprendidas la posicionaron como una universidad de vanguardia en esta temática, en gran medida gracias al apoyo de la Rectora de la Universidad, quien por estos años ha sido prácticamente la única rectora mujer al frente de una universidad pública en el país. 


\title{
Discusión
}

\section{\#El silencio marca tu vida o los caminos de la praxis}

Hasta aquí he descrito la realidad que en materia de acoso y hostigamiento sexual insiste y persiste en las universidades mexicanas y particularmente lo que acontece en la Veracruzana, mi universidad de adscripción. Así mismo, las acciones emprendidas para erradicar este fenómeno. Lo que a continuación presentaré, es un análisis de todo este proceso de trabajo y de sus resultados, lo que concibo como un trayecto de reflexión personal, institucional y político, en tanto se trata de una praxis que me ha implicado activamente como investigadora, como profesora y como mujer.

Concuerdo con Leticia Cufré Marchetto (2017, p. 52) cuando afirma que es "particularmente valiosa la reflexión sobre nuestra implicación y exploración acerca del espacio o localización óptima como investigadores respecto a lo investigado, sobre todo cuando, como sucede con las violencias, la problemática que abordamos es potencialmente implicante". Ello, siguiendo a Bourdieu (1980) conlleva, necesariamente, a una "pérdida de la distancia objetivante", situación que nos aparece, continúa Cufré Marchetto (2017, p. 52):

\begin{abstract}
como las dos caras de una moneda: una que expresa la preocupación y empatía y, otra en la que suele estar el temor frente al riesgo de quedar atrapados por lo que quien ocupe el rol de investigador tendrá una función muy similar a la del testigo donde ambos, investigador y testigo, sufrirán permanentemente las tensiones de estar demasiado cerca o demasiado lejos y, sobre todo, tendrán que soportar la sensación de que siempre se llega tarde.
\end{abstract}

Y todo ello porque "las violencias atacan la capacidad de pensar a falta de disposiciones éticas o políticas y también ante la falta de instrumentos de pensamiento tales como problemáticas, conceptos, métodos, técnicas” (CUFRÉ MARCHETTO, 2017, p. 52).

Vuelvo también al texto clásico titulado: "De la ansiedad al método en las ciencias del comportamiento" (DEVEREUX, 1994), porque la metodología empleada para este trabajo partió necesariamente de mi propia ansiedad frente a los casos de acoso y hostigamiento sexual sufridos por mis compañeras de trabajo, por estudiantes, por trabajadoras de la universidad, por esa angustia experimentada de manera permanente frente a estas situaciones en un contexto particularmente violento en mi país y en el Estado de Veracruz ${ }^{\text {iv }}$. Sucesos tan difíciles como secuestros, desapariciones y asesinatos de miembros de la comunidad universitaria y de sus familias mismos que simultáneamente me llenaban de indignación y me hacían sentir a veces demasiado cerca del objeto de estudio motivándome a la acción, a salir de la parálisis y a pensar en nuevas formas de intervención a fin de no ubicarme como un mero testigo y, por ende demasiado lejos del mismo. Fui caminando a ciegas pero siguiendo los buenos consejos de Sigmund Freud, quien solía decir que cuando no tengamos luz hemos de aprender a ver mejor en la oscuridad.

Y porque ciertamente investigar es desentrañar la realidad a través de procesos de subjetivación de lo cotidiano, el quehacer ahora narrado parte de mi propia subjetividad en tanto praxis que me ha llevado de la teoría a la práctica y viceversa, con la esperanza de generar, 
en el mejor de los casos, una espiral virtuosa de conocimiento transformador. En este sentido, al asumir la Coordinación de la Unidad de Género recién terminaba de escribir un libro sobre las mujeres titulado: "Las mujeres y su goce: del silencio al lenguaje fecundo" que se publicó en 2015. Venía pues, de recorrer un camino de teorización y ahora me movía hacia otro de práctica cotidiana a fin de apoyar a las universitarias a transitar, junto conmigo, del silencio impuesto al lenguaje emancipador, tal como lo había descrito a lo largo de mi obra recién concluida. Y por fortunas del feminismo, como bien lo denomina (FRASER, 2015) referido a las acciones colectivas emprendidas por las mujeres, el trabajo que llevé a cabo en favor de la igualdad de género y por una vida académica libre de violencia, contó con la participación de muchos hombres y mujeres de la comunidad universitaria unidos bajo el lema "\#elsilenciomarcatuvida".

\section{¿Podremos erradicar la violencia contra las mujeres?}

Al analizar los movimientos de mujeres del siglo XX, Touraine (1997), se preguntaba si al final del día hombres y mujeres, iguales y diferentes desde su perspectiva - aunque también pueden concebirse como iguales y/o diferentes (LAURENT-ASSOUN, 1998), e incluso radicalmente diferentes (IRIGARAY, 1999), - podrían vivir juntos. La la pregunta parte del hecho de que la acción colectiva de las mujeres desde su perspectiva, tiene como punto de partida la lucha por la identidad, es decir, parte del análisis de la condición y la posición de las mujeres en el mundo concebidas como iguales en derechos que los varones, pero diferentes en sus posibilidades de acceso a un mundo igualitario y justo, y es que los mayores obstáculos a los que se han enfrentado las políticas de género y que han implicado la lucha constante por su propia legitimidad, es que, como lo analiza Touraine, se perciben como acciones que amenazan a la unidad del colectivo social. Sin embargo, dado que se trata de un falso colectivo humano que se ha establecido desde el genérico universal "hombre", ello es lo que ha motivado a millones de mujeres a ejercer una acción colectiva reivindicando sus diferencias y evidenciando la riqueza de la vida social en la diversidad.

Ellas han situado el problema en las relaciones asimétricas de poder que hacen que las diferencias se tornen en desigualdades, producto de estructuras sociales ancladas en el poder hegemónico de dominación masculino, perpetuado durante siglos por grupos privilegiados de varones, incluidas también algunas mujeres. El llamado unánime ha sido hacia el empoderamiento y al acceso a una vida libre de violencia de todas las mujeres y, particularmente, de quienes viven en condiciones de mayor despojo y desprotección en quienes se intersectan condiciones de pobreza, etnia y clase.

Ciertamente la universidad no ha sido ajena al impacto de estos movimientos sociales. Considero que todo ello nos ha llevado a que hoy en día las académicas nos movamos también en las aguas movedizas de la identidad, es decir, en ese tipo de lugar desde donde nos cuestionamos permanentemente quiénes vamos siendo, cómo vivir juntos sin invisibilizarnos y cómo devenir sujetos de nuestro propio deseo. Y desde este lugar es claro que el fenómeno de la violencia e identificar sus causas es un asunto muy complejo, y coincido al respecto con en que la violencia es producto de la convivencia humana y por ello es posible revertirla 
también desde la acción humana, lo que implica que los diversos grupos sociales desarrollen normas y acuerdos colectivos que fomenten el desarrollo de vínculos humanos no destructivos. En tanto el ser humano es un ser de posibilidades, la violencia no está genéticamente determinada sino que se desarrolla en función de lo que se vive, de la conciencia y el aprendizaje de la experiencia que se va adquiriendo a lo largo de la existencia sobre aquello que se puede y quiere modificar con respecto a sí mismo.

La violencia de género al ser producto de estructuras sociales androcéntricas, patriarcales y de dominación masculina que se traducen en condiciones de vida, de acceso de oportunidades y de derechos humanos inequitativas y desiguales entre hombres y mujeres, y que se reproduce mediante procesos de naturalización, normalización y normatización social, es decir, que se impone a las mujeres por el mero hecho de serlo y se mantiene y perpetúa gracias al despliegue de macro y micro formas de dominio cotidiano, de mecanismos de vigilancia, control y castigo (FOUCAULT, 2015), es, sin embargo, susceptible de ser desmontada mediante el accionar colectivo a través de procesos de búsqueda de redistribución económica, reconocimiento social y de representación política (FRASER, 2015).

Erradicar la violencia de género en todos los ámbitos de convivencia humana, y particularmente en la universidad, nos convoca a emprender acciones transformadoras y simultáneas a nivel global, pero en sintonía con las necesidades locales y en los distintos espacios donde actúan las mujeres, buscando ser remuneradas y participar en el desarrollo económico en igualdad de condiciones que nuestros pares varones; estar bien representadas en todas las instancias de toma de decisión donde se dirimen los temas que nos atañen y afectan $\mathrm{y}$, ser reconocidas por nuestras aportaciones a la vida social y colectiva, no sólo en términos de "meritocracia" por ejercer liderazgos individuales o colectivos, sino también por visibilizar los aportes cotidianos a la vida reproductiva y al cuidado de los demás. El espacio universitario es un entorno privilegiado para el cambio y el reconocimiento de las desigualdades, en tanto le corresponde a la universidad ejercer el poder de la transformación y la conciencia.

\section{La apuesta por la palabra generadora de sentido.}

Frente al acto violento, destructivo y que anula al sujeto se impone la palabra generadora de sentido, esa que devuelve a las personas su calidad de sujeto actor, capaz de construirse a sí mismas y de transformar su entorno generando consensos y acuerdos con los demás para vivir civilizadamente, es decir, para convivir regidos por el bien común. Sin embargo, los intercambios sociales que imperan en nuestra sociedad son justamente lo contrario, puesto que el otro no se concibe como sujeto y ello lo convierte en objeto despojándolo de su capacidad de palavra, lo que lo condena al silencio. Colocado ya sin voz, en una posición de subordinación, se ejerce sobre él o ella la violencia, es decir, se le imponen acciones, lenguajes, palabras mediante mecanismos coercitivos, inducidos o forzados.

Aunado a ello, hay que recalcar que nuestras sociedades se han construido en base a un sistema socioeconómico y cultural monosubjetivo que se rige bajo el modelo humano masculino y, por ello, el lenguaje que funciona como uno de los principales medios de producción de sentido y rige los códigos de las relaciones sociales, en lugar de ser comunicativo 
a fin de establecer el diálogo entre dos sujetos, se torna impositivo funcionando como un medio para transmitir información a un otro que no es considerado como tal, lo que impide el intercambio entre seres diferentes que crean juntos nuevas significaciones.

La apuesta por la palabra generadora de sentido es pues, la apuesta por el sujeto humano dialógico, por el intercambio entre las personas en su diversidad y se sustenta en el reconocimiento del otro en tanto tal a fin de construir colectivamente entornos civilizatorios. El lenguaje dialógico se concibe como una construcción humana que regula y genera sentido social. La convivencia social requiere entonces el que las sociedades reconozcan la capacidad de las personas de comunicarse y de generar los procesos de regulación necesarios que permitan el intercambio fecundo. Aquello que permite eliminar todas las formas de violencia entre las personas y los grupos sociales es entonces, siguiendo a Bleichmar (2009) el potenciar un proceso de recomposición de las fracturas sociales que permitan normar la convivencia humana de ciudadanas y ciudadanos que son sujetos, agentes de su propia vida y que se comunican y relacionan entre si bajo nexos de solidaridad y trabajo compartidos.

De esta manera, terminar con la violencia de género pasa necesariamente por la refundación de los diversos lenguajes humanos: el lenguaje jurídico, el lenguaje gramatical, el lenguaje artístico, por nombrar sólo algunos de ellos. Implica poner al centro del debate y la acción colectiva la formulación de instrumentos jurídicos institucionales con perspectiva de género, la incorporación del lenguaje incluyente en el quehacer universitario y la generación de propuestas innovadoras de intercambio y transmisión de conocimiento con perspectiva de género entre las y los actores universitarios. Y, desde luego, la escucha activa y el acompañamiento a las víctimas a fin de sacarlas del lugar del silencio en que han sido colocadas para que sean capaces de denunciar la violencia sufrida e iniciar los procesos de impartición de justicia correspondientes.

En relación a las denuncias de acoso y hostigamiento sexual que recientemente se han hecho visibles a través de movimientos como \#metoo, \#cuentatuhistoriadeacoso, etc, se han generado muchos debates acerca de la veracidad de este tipo de denuncias. Al respecto, autoras como Lamas (2018), reconocida feminista mexicana, han comenzado a cuestionar si el acoso sexual ha devenido tan mediático que se ha prestado a actos victimizantes más que a denuncias reales, producto de actitudes revanchistas y de una concepción puritana de la sexualidad. Sin duda, esta cuestión requiere de un análisis amplio que excede los límites de este trabajo, pero no quisiera dejar de señalar que las relaciones eróticas entre las personas no pueden colocarse únicamente en el lugar del deseo, si bien siempre presente en nuestra vida y aun cuando escapa inevitablemente a nuestra voluntad. Considero, como lo he venido argumentando, que todas las interacciones humanas requieren ser reguladas por mecanismos culturales mismos que, si bien generan malestar (FREUD, 1929) son los que garantizan la sana convivencia social, porque sabemos que aquello que acontece a nivel micro-social, como lo es la relación sexual entre las personas, incluso cuando aparezca como un acto consensuado, es también un acto social que está reproduciendo la desigualdad, las jerarquías de poder, la anulación del sujeto y la enajenación macro social imperante. Por ello, en nuestro caso, en la UV atendimos a todas las personas que consideraron haber sido objeto de abuso y hostigamiento sexual y nos aseguramos que contaran con los mecanismos institucionales necesarios para emprender un 
proceso de impartición de justicia y de reparación del daño si elegían hacerlo. Cuando así lo decidieron, les acompañamos en los procesos de denuncia siguiendo los mecanismos institucionales existentes.

A la par, generamos múltiples espacios de diálogo entre las y los diversos actores de la vida universitaria mediante talleres, cursos, seminarios, elaboración de videos, de obras artísticas, de mensajes enviados por medios electrónicos y redes sociales, entre otras múltiples estrategias implementadas conjuntamente para el intercambio fecundo de ideas a partir de las cuales surgieron las acciones emprendidas para la prevención y erradicación de la violencia de género en nuestra universidad.

Finalmente, si bien es cierto que no logramos todo aquello que nos propusimos, fuimos capaces de mantenernos en nuestro lugar de sujetos que reconocen a los otros como tales y, por ende, en el lugar de investigadores que investigan para desentrañar la realidad a través de procesos de subjetivación de lo cotidiano, es decir, desde una praxis que contribuye al proceso civilizatorio de creación de un lenguaje científico capaz de construir nuevas realidades.

\section{Referencias}

BLEICHMAR, Silvia. Violencia social, violencia escolar. Argentina: Noveduc, 2009.

BOURDIEU, Pierre. EI Sentido práctico. España: Taurus Humanidades, 1980.

CASILLAS, Miguel et al. Tipos de violencia en la Universidad: Primeros resultados de una investigación en la UV. In: CASILLAS, Miguel Angel; DORANTES, Jesyra; ORTIZ, Verónica (coord.) Estudios sobre violencia de género en la universidad. México: Biblioteca Digital de Humanidades. 2017. p. 55-78.

CEDAW. Informe Sombra sobre el seguimiento de las recomendaciones del Comité CEDAW al Estado Mexicano. 2018. Recuperado en: encurtador.com.br/dfxSW. Acceso el: 6 Nov. 2018.

CUFRÉ MARCHETTO, Leticia. Investigar prácticas sociales violentas. In: CASILLAS, Miguel Angel; DORANTES, Jesyra; ORTIZ, Verónica (coord.) Estudios sobre violencia de género en la universidad. México: Biblioteca Digital de Humanidades. 2017. p. 40-51.

DEVEREUX, Georges. De la ansiedad al método en las ciencias del comportamiento. México. Siglo XXI. 1994.

FOUCAULT, Michel. Vigilar y castigar: nacimiento de la prisión. México: Siglo XXI, 2015.

FRASER, Nancy. Fortunas del Feminismo. Argentina: Traficantes de sueños. 2015.

FREUD, Sigmund. El malestar en la cultura, México: Ed. Kindle, 1929. 
GARCÍA, María José (coord.) Igualdad Sustantiva en las Instituciones de Educación Superior. México: Universidad Veracruzana, Dirección Editorial, 2018.

GUZMÁN IBÁÑEZ, María de Lourdes Lidia. Atención y prevención del acoso sexual. In: CASILLAS, Miguel Angel; DORANTES, Jesyra; ORTIZ, Verónica (coord.) Estudios sobre violencia de género en la universidad. México: Biblioteca Digital de Humanidades. 2017. p. 88-97.

IRIGARAY, Luce. Ser dos. España: Paidós Ibérica, 1999.

JONGITUD ZAMORA, Jaqueline. Discriminación por identidad de género: propuesta para su prevención y erradicación em instituciones de educación superior. In: CASILLAS, Miguel Angel; DORANTES, Jesyra; ORTIZ, Verónica (coord.) Estudios sobre violencia de género en la universidad. México: Biblioteca Digital de Humanidades. 2017. p. 169-188.

LAMAS, Marta. Acoso ¿denuncia legítima o victimización? México: Cenzontle, Fondo de Cultura Económica, 2018.

LAURENT ASSOUN, Paul. El perverso y la mujer. Argentina: Nueva Visión, 1998.

MEXICO. Ley General de Acceso de las Mujeres a una Vida libre de Violencia. Cámara de Diputados Del H. Congreso de la Unión. 2018. Recuperado en: http://www.diputados.gob.mx/LeyesBiblio/ref/lgamvlv.htm. Acceso el: 20 Nov. 2018.

OCDE. En México, de cada 100 niños que entran a la primaria, solo 21 terminan la Universidad. Ciudade de México, 23 agosto de 2018. Recuperado en:

http://www.articulosiete.com.mx/en-mexico-de-cada-100-ninos-que-entran-a-la-primariasolo-21-terminan-la-universidad-ocde/. Acceso el: 20 Nov. 2018.

ONU. Mujeres. Status of women in science and technology. $55^{\mathrm{a}}$ sesión, febrero 22 a marzo 4, Nueva York. 2011

TOURAINE, Alain. ¿Podremos vivir juntos?, iguales y diferentes. Madrid: PPC Editorial. 1997.

UNIVERSIDAD VERACRUZANA. Guía para la atención de casos de acoso y

hostigamiento sexual. México: Coordinación de la Unidad de Género de la Universidad Veracruzana. 2015a.

UNIVERSIDAD VERACRUZANA. Reglamento para la igualdad de género de la Universidad Veracruzana. México: Universidad Veracruzana. 2015b. Recuperado en: https://www.uv.mx/legislacion/files/2015/12/Reglamento-para-la-Igualdad-de-Genero.pdf. Acceso el: el 20 de Noviembre del 2018.

\section{Notas}

\footnotetext{
${ }^{\mathrm{i}}$ Recuperado en:

https://www.uv.mx/prensa/general/violencia-contra-las-mujeres-aun-persiste/. Acceso el: 20 Nov. 2018.

ii México es el país del mundo con la mayor tasa de embarazo adolescente. De acuerdo a la CEDAW (2018, p. 7-

8), "el matrimonio infantil se encuentra directamente relacionado con el embarazo adolescente y la violencia
} 
sexual. En 2015, la cifra de nacimientos en madres de 10 a 17 años fue de 144,642, de ese total los nacimientos de madres adolescentes de entre 12 y 14 años nacieron 5,586 niñas y niños. Cifra aún más alarmante son los 54 nacimientos de madres niñas de entre 9 y 11 años. Las uniones conyugales, los embarazos y nacimientos en madres menores de 18 años son un problema nacional [...] Esta situación significa para muchas mujeres la causa de abandono escolar y por tanto repercute en su desarrollo personal y social".

iii Para ahondar sobre esta temática consultar García Oramas (2018). Igualdad Sustantiva en las Instituciones de Educación Superior. Versión digital en: http://libros.uv.mx/index.php/UV/catalog/book/TU178. Acceso el: 20 Nov. 2018.

iv Según cifras oficiales, desde 2007 en México hay alrededor de 34.500 desaparecidos por la guerra contra el narcotráfico, de los cuales únicamente .9\% han sido identificados, es decir alrededor de 340 casos (Secretaria de Gobernación, 2018); existen alrededor de 250 fosas clandestinas que han sido halladas por colectivos de búsqueda de desaparecidos solo en el Estado de Veracruz, con restos humanos aun no identificados (Diario La Jornada, 2018) y, anualmente, acontecen en México 2,500 feminicidios y de ellos tan solo en Veracruz se han dado 117 casos y 125 mujeres continúan desaparecidas desde 2013, mientras sólo en lo que va del año en curso, 2018, se han registrado 78 feminicidios (Observatorio Ciudadano Nacional del Feminicidio (OCNF). 